\title{
Synthesis of cerium hydroxycarbonate powders via a hydrothermal technique
}

\author{
Hsien-Cheng Wang, Chung-Hsin Lu* \\ Department of Chemical Engineering, National Taiwan University, Taipei 106, Taiwan, ROC \\ (Refereed) \\ Received 6 July 2001; accepted 7 September 2001
}

\begin{abstract}
$\mathrm{CeOHCO}_{3}$ powders have been directly synthesized using a hydrothermal process at temperatures as low as $160^{\circ} \mathrm{C}$. The well-dispersed powders are obtained in a short period of reaction time during hydrothermal reaction via the hydrolysis of urea. For synthesizing $\mathrm{CeOHCO}_{3}$, the concentration of urea is found to be a crucial determinant, which has significant effects on the morphology of the derived powders. When low urea concentrations are provided, the formed particles are rhomboidal platelets. On the other hand, the high urea concentrations cause the shape of the powders to become prismatic. Increasing the concentration of urea tends to increase the particle size as well as the aspect ratio of $\mathrm{CeOHCO}_{3}$ powders. After further heating at $500^{\circ} \mathrm{C}$, a phase transformation from orthorhombic $\mathrm{CeOHCO}_{3}$ to cubic $\mathrm{CeO}_{2}$ takes place. The crystallinity and size of $\mathrm{CeO}_{2}$ strongly depend on the particle size of $\mathrm{CeOHCO}_{3}$. (C) 2002 Elsevier Science Ltd. All rights reserved.
\end{abstract}

Keywords: A. Ceramics; A. Inorganic compounds; B. Chemical synthesis; C. X-ray diffraction

\section{Introduction}

In general, ideal ceramic powders should meet certain criteria, for example, uniform morphology, well-controlled chemical composition, narrow size distribution, and high crystallinity [1-3]. For preparing ideal ceramic powders, different types of solution processes such as precipitation method, sol-gel process and hydrolysis of alkoxides have been intensively investigated [4-6]. In order to synthesize well-

\footnotetext{
* Corresponding author. Tel.: +886-2-2363-5230; fax: +886-2-2362-3040.

E-mail address: chlu@ccms.ntu.edu.tw (C.-H. Lu).
} 
crystallized powders, calcining the precursors obtained from the solution processes at high temperature is necessary. Nevertheless, the high temperature calcination induces serious grain growth of ceramic powders, which will reduce the sinterability of the obtained powders. On the other hand, among all solution process, the hydrothermal method shows great potential in directly achieving the crystallized ceramic powders from the solution, without further calcinations [7-10]. The hydrothermal process not only shortens the required reaction time, but also lowers the temperature for synthesizing ceramic powders. With the above benefits, the hydrothermal process can significantly reduce the energy consumed and decrease the particle size of the powders obtained [11-13].

In the previous studies in which the conventional precipitation method is adopted, crystallized cerium hydroxycarbonate $\left(\mathrm{CeOHCO}_{3}\right)$ powders are obtained by aging the starting solution for tens of hours [14-16]. In addition, the starting solution containing the cerium cations is limited to certain low concentration ranges, and the yield of powders is low. For reducing the above drawbacks, a new hydrothermal process is introduced in this study to shorten the reaction time and increase the yield of $\mathrm{CeOHCO}_{3}$ powders. Urea is selected as the alkali source for synthesizing $\mathrm{CeOHCO}_{3}$. The effects of urea concentration on the morphology of crystallized $\mathrm{CeOHCO}_{3}$ powders are mainly investigated. The phase transformation from $\mathrm{CeOHCO}_{3}$ to $\mathrm{CeO}_{2}$ is also examined.

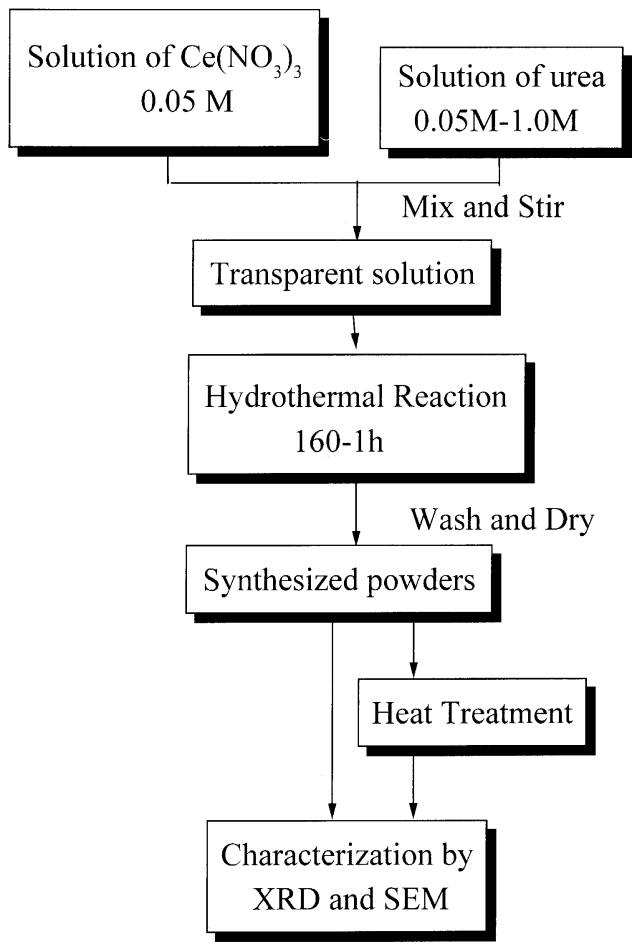

Fig. 1. Schematic diagram for the synthesis of $\mathrm{CeOHCO}_{3}$ powders by the hydrothermal technique. 


\section{Experimental}

The synthesis procedures of $\mathrm{CeOHCO}_{3}$ and $\mathrm{CeO}_{2}$ powders using the hydrothermal technique are illustrated by the flow chart shown in Fig. 1. Cerium nitrate hexahydrate, $\mathrm{Ce}(\mathrm{NO})_{3} \cdot 6 \mathrm{H}_{2} \mathrm{O}$ and urea, $\mathrm{CO}\left(\mathrm{NH}_{2}\right)_{2}$ were adopted as the starting materials. Cerium(III) salt solution $(0.05 \mathrm{M})$ and urea solutions of various concentrations $(0.05$, $0.1,0.3$, and $1.0 \mathrm{M}$ ) were mixed and hydrothermally reacted for $1 \mathrm{~h}$. These reagent grade reactants were first individually dissolved in deionized water at room temperature and then mixed thoroughly. The mixed solutions were introduced into a Teflon-lined autoclave apparatus and hydrothermally reacted at $160^{\circ} \mathrm{C}$ for $1 \mathrm{~h}$. The hydrothermal apparatus was equipped with a mechanical stirrer rotating at a speed of $200 \mathrm{rpm}$. After hydrothermal reaction, the reactor was cooled to ambient temperature. The prepared powders were washed repeatedly with distilled water and alcohol to remove the adsorptive ions and then dried at $80^{\circ} \mathrm{C}$ in air. The structural characterization was carried out by the X-ray powder diffraction using the Ni-filtered $\mathrm{Cu} \mathrm{K} \alpha$ radiation. The particle size and morphology of the powders were examined via a scanning electron microscope (SEM).

\section{Results and discussion}

The XRD results of hydrothermally derived powders at $160^{\circ} \mathrm{C}$ are shown in Fig. 2 . This figure reveals that the powders obtained under all conditions exhibit highly developed crystallinity. When the concentration of urea is $\geq 0.1 \mathrm{M}$, the XRD patterns are similar to that reported in ICDD file [17], indicating the successful fabrication of monophasic $\mathrm{CeOHCO}_{3}$ powders through the hydrothermal reaction. The obtained $\mathrm{CeOHCO}_{3}$ powders exhibit an orthorhombic structure. It is also found that increasing the concentration of urea leads to improved crystallinity of $\mathrm{CeOHCO}_{3}$ powders. In comparison with the previous precipitation method [14-16], this hydrothermal process is confirmed to markedly shorten the reaction time for synthesizing $\mathrm{CeOHCO}_{3}$ powders. As for the reaction at a low concentration of urea $(0.05 \mathrm{M})$, a small amount of $\mathrm{CeO}_{2}$ is found to exist with $\mathrm{CeOHCO}_{3}$. It is considered that there is not enough carbonate ions for all cerium cations to form $\mathrm{CeOHCO}_{3}$ at low concentration of urea.

Fig. 3 illustrates the microstructures of hydrothermally prepared $\mathrm{CeOHCO}_{3}$ powders. This figure indicates that the morphology of the obtained powders is strongly influenced by the concentration of urea. When urea concentration equals $0.05 \mathrm{M}$, angular $\mathrm{CeOHCO}_{3}$ particles with a size of 3-4 $\mu \mathrm{m}$ are formed as shown in Fig. 3(a). When the urea concentration is $0.1 \mathrm{M}$, the morphology of $\mathrm{CeOHCO}_{3}$ changes to rhomboidal platelets with an aspect ratio of 1.3-1.5 and a length of 6-7 $\mu \mathrm{m}$ in the long axis (see Fig. 3(b)). The aspect ratio of particles is defined as the ratio of the length in the long axis to that in the short axis. Once the urea concentration further rises to $1 \mathrm{M}$, $\mathrm{CeOHCO}_{3}$ particles with a prismatic shape are formed, and the length in the long axis and the aspect ratio of the particles increase to 12-13 and 3.0-3.5 $\mu \mathrm{m}$, respectively (see 


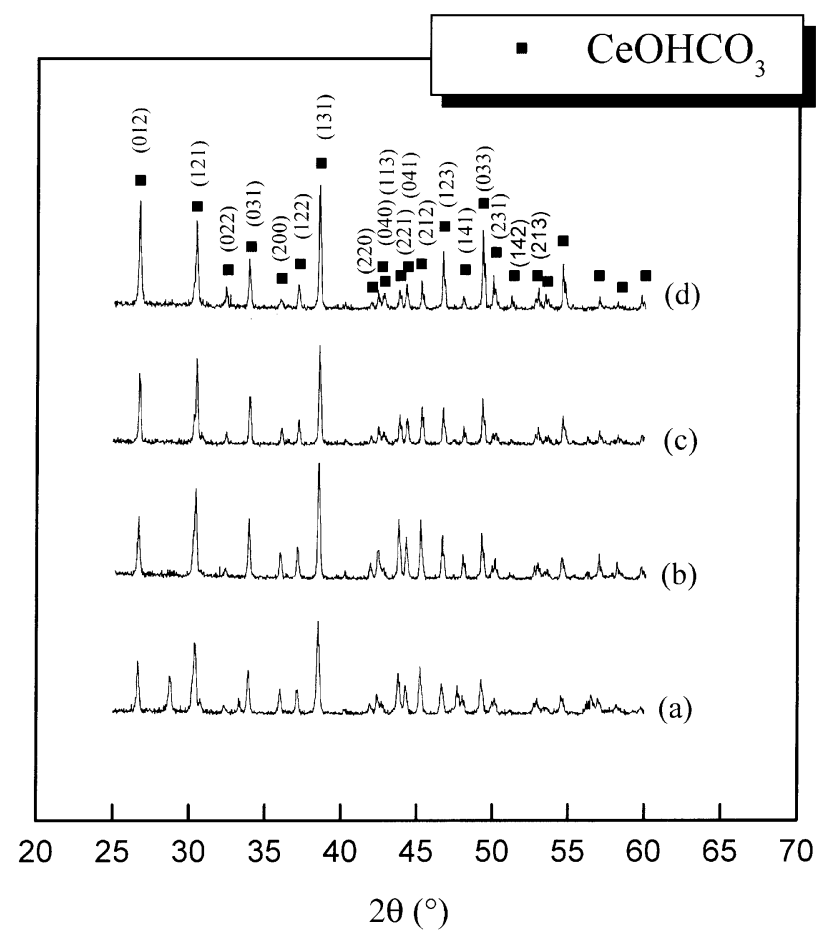

Fig. 2. Room temperature XRD patterns of the hydrothermally derived powders at urea concentrations equal to (a) $0.05 \mathrm{M}$; (b) $0.1 \mathrm{M}$; (c) $0.3 \mathrm{M}$; (d) $1.0 \mathrm{M}$.

Fig. 3(c)). Increasing urea concentration is found to significantly increase the particle size and the aspect ratio of $\mathrm{CeOHCO}_{3}$ particles. With the hydrolysis of urea at high concentration, the newly derived nuclei of $\mathrm{CeOHCO}_{3}$ crystallites tend to flock and conglomerate with the previously formed particles, rather than to form new nuclei, thereby resulting in the morphological change and the grain growth.

The $\mathrm{pH}$ values measured before and after the hydrothermal reactions are illustrated in Fig. 4. Prior to the hydrothermal reaction, the $\mathrm{pH}$ value of the unreacted solution slightly increases with a rise in the urea concentration. After the hydrothermal reaction at low urea concentration $(0.05 \mathrm{M})$, the $\mathrm{pH}$ value of the reacted solution becomes lower than that of the original (unreacted) solution. However, at urea concentration $\geq 0.1 \mathrm{M}$, the $\mathrm{pH}$ value of the reacted solution after the hydrothermal reaction is much higher than that of the original (unreacted) solution. The hydroxylation processes of metal ions have been studied by Matijevic [18]. According to their investigation, deprotonation of hydrated metal ions is accelerated at elevated temperatures. The deprotonation of hydrated cerium cations is considered as below:

$$
n\left[\mathrm{Ce}\left(\mathrm{H}_{2} \mathrm{O}\right)_{p}\right]^{3+} \rightarrow\left[\mathrm{Ce}_{n}\left(\mathrm{H}_{2} \mathrm{O}\right)_{n p-m}(\mathrm{OH})_{m}\right]^{(3 n-m)+}+m \mathrm{H}^{+}
$$

Once protons are released into the solution, the $\mathrm{pH}$ value of the solution will be lowered after the hydrothermal reaction. On the other hand, when urea concentration increases, a large amount of $\mathrm{CO}_{3}{ }^{2-}$ and $\mathrm{OH}^{-}$are produced after the hydrolysis of 


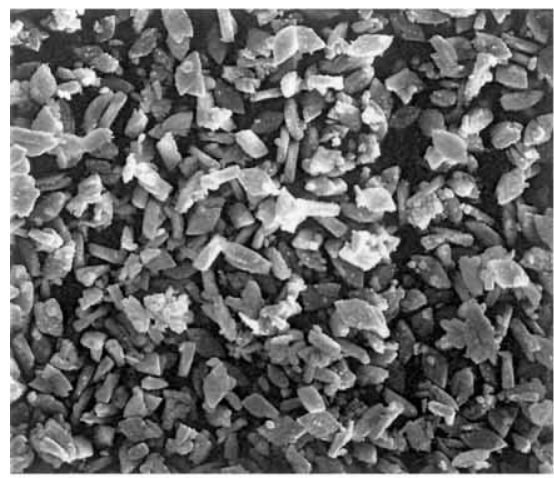

(a)
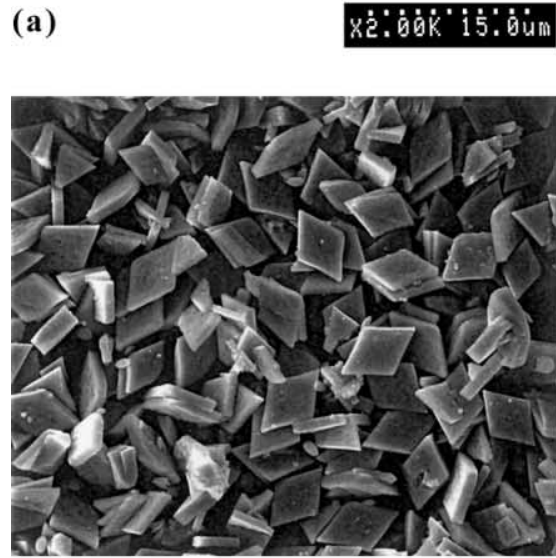

(b)

хอ่:ต่อ่์

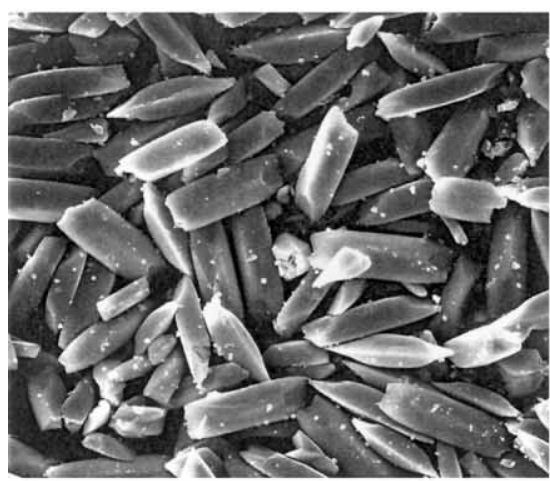

(c)

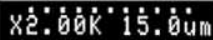

Fig. 3. Scanning electron micrographs of hydrothermally derived $\mathrm{CeOHCO}_{3}$ powders at urea concentrations equal to (a) $0.05 \mathrm{M}$; (b) $0.1 \mathrm{M}$; (c) $1.0 \mathrm{M}$.

urea. These carbonate and hydroxide ions will react with $\mathrm{Ce}^{3+}$ cations to form $\mathrm{CeOHCO}_{3}$ according to Eq. (2)

$$
\mathrm{Ce}^{3+}+\mathrm{CO}_{3}^{2-}+\mathrm{OH}^{-} \rightarrow \mathrm{CeOHCO}_{3}
$$




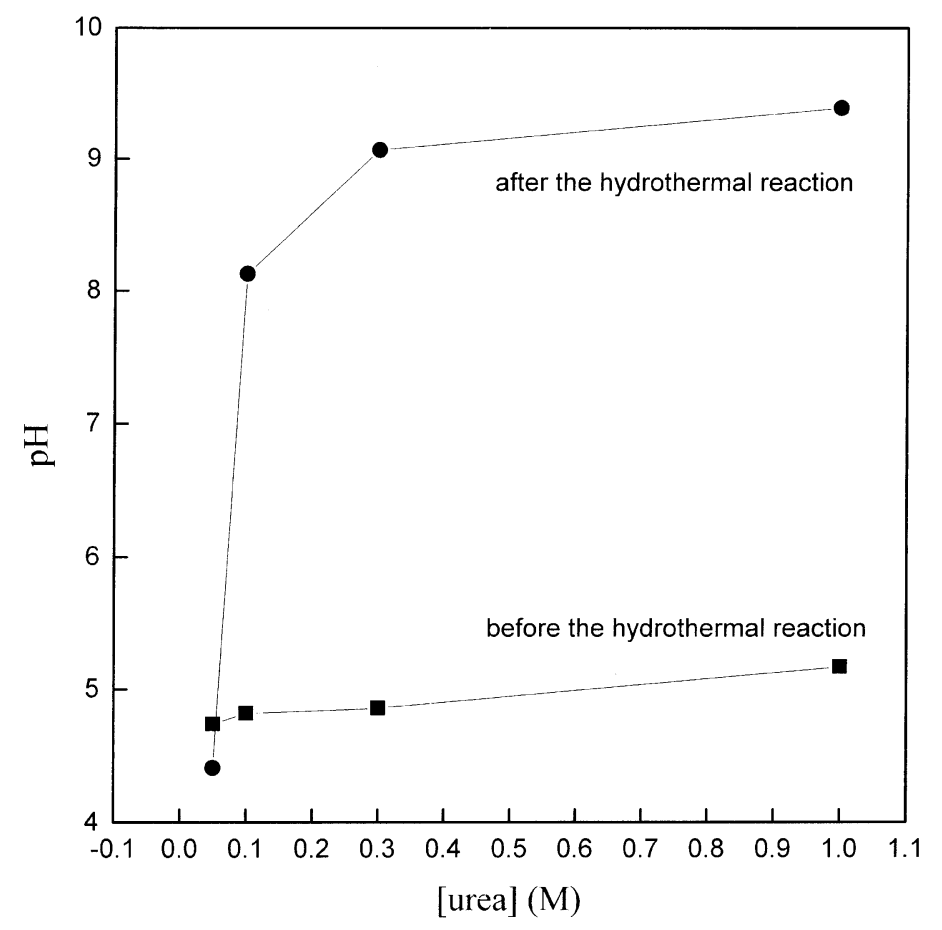

Fig. 4. The $\mathrm{pH}$ values of the solutions before and after the hydrothermal reactions.

One mole can produce $2 \mathrm{~mol}$ of hydroxide ions and $1 \mathrm{~mol}$ of carbonate ions after the hydrolysis of urea. However, during the formation of 1 mol of $\mathrm{CeOHCO}_{3}$, only 1 mol of hydroxide ions and $1 \mathrm{~mol}$ of carbonate ions are consumed; therefore, excess hydroxide ions are left in the solution, thereby raising the $\mathrm{pH}$ value of the reacted solution.

Fig. 5 depicts the relation between the yield of the products and urea concentrations. When urea concentration equals $0.05 \mathrm{M}$, the yield is only $66 \%$. Increasing the urea concentration markedly increases the yield of the powders. At urea concentration equal to $1 \mathrm{M}$, most $\mathrm{Ce}^{3+}$ reacts with carbonate and hydroxide ions and consequently the yield of $\mathrm{CeOHCO}_{3}$ reaches around $95 \%$. The yield of products and the $\mathrm{pH}$ value of the reacted solution respond in similar ways to urea concentration. This implies that the formation of $\mathrm{CeOHCO}_{3}$ is mainly dominated by the hydrolysis of urea.

The obtained $\mathrm{CeOHCO}_{3}$ powders were further heated at $500^{\circ} \mathrm{C}$ for $1 \mathrm{~h}$, and the corresponding XRD patterns are shown in Fig. 6. After the heat treatment, all specimens transform into pure $\mathrm{CeO}_{2}$ powders with a cubic structure [19]. The phase transformation of $\mathrm{CeOHCO}_{3}$ into $\mathrm{CeO}_{2}$ after the heat treatment can be elucidated by the following equation:

$$
4 \mathrm{CeOHCO}_{3}+\mathrm{O}_{2} \rightarrow 4 \mathrm{CeO}_{2}+4 \mathrm{CO}_{2}+2 \mathrm{H}_{2} \mathrm{O}
$$

The crystallinity of $\mathrm{CeO}_{2}$ powders is found to depend on the preparation conditions of $\mathrm{CeOHCO}_{3}$. When a low urea concentration is used for preparing $\mathrm{CeOHCO}_{3}$, the crystallinity of $\mathrm{CeO}_{2}$ powders increases. 


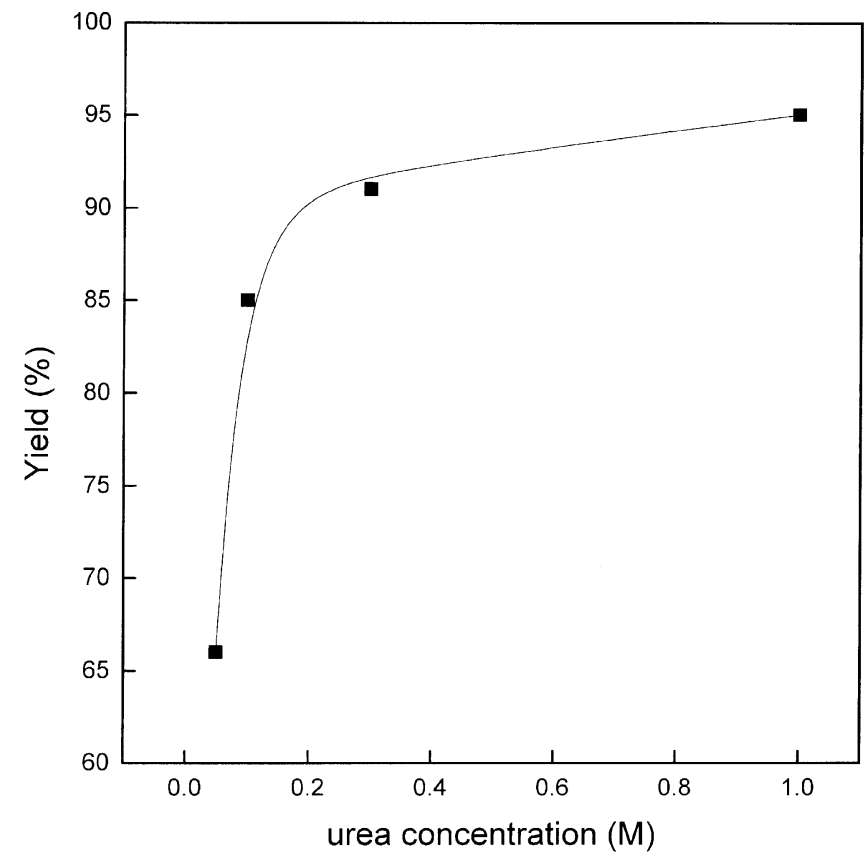

Fig. 5. The relation of the yield of the hydrothermally derived powders vs. urea concentration.

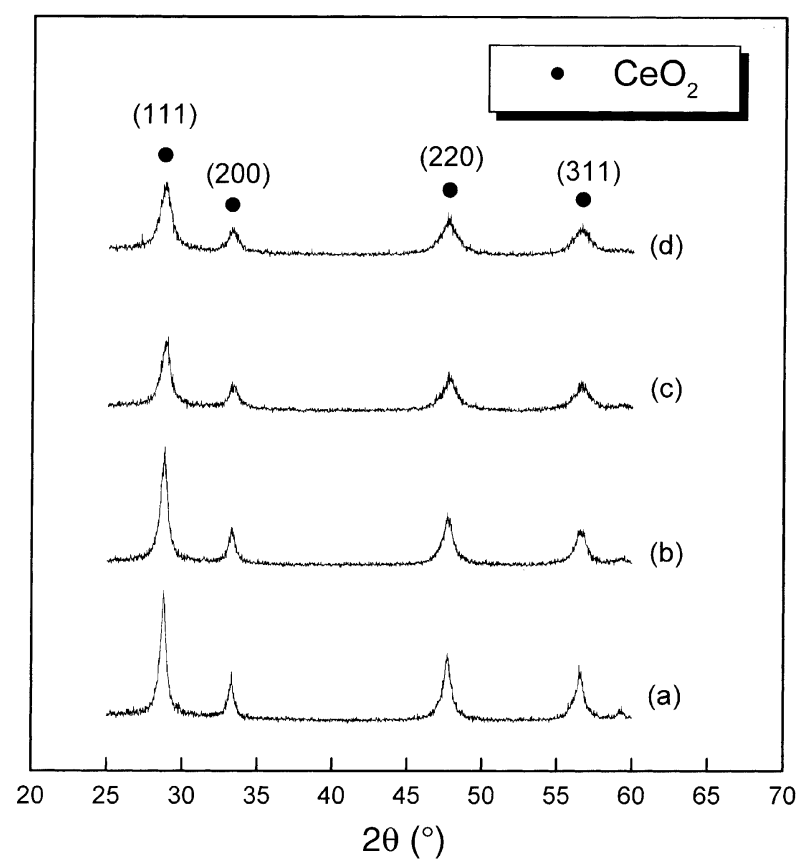

Fig. 6. XRD patterns of the $\mathrm{CeO}_{2}$ powders obtained by heating $\mathrm{CeOHCO}_{3}$ powders which are prepared using urea concentrations equal to (a) $0.05 \mathrm{M}$; (b) $0.1 \mathrm{M}$; (c) $0.3 \mathrm{M}$; (d) $1.0 \mathrm{M}$. 
The microstructures of $\mathrm{CeO}_{2}$ powders derived from $\mathrm{CeOHCO}_{3}$ are shown in Fig. 7. The morphology of $\mathrm{CeO}_{2}$ powders is found to maintain the original shape of $\mathrm{CeOHCO}_{3}$ after the heat treatment. When urea concentration rises from 0.05 to $1 \mathrm{M}$, the morphology of $\mathrm{CeO}_{2}$ powders changes from a rhomboidal shape to a

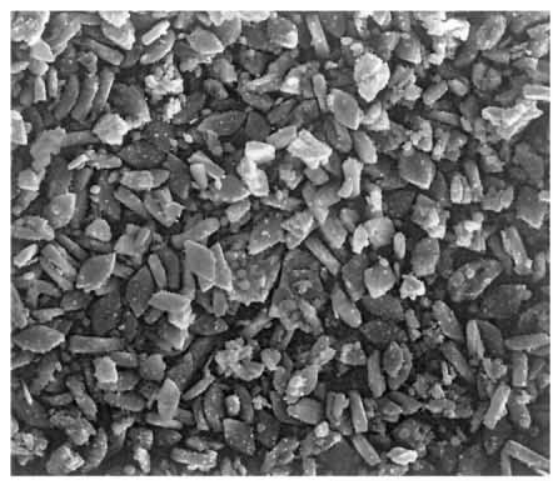

(a)
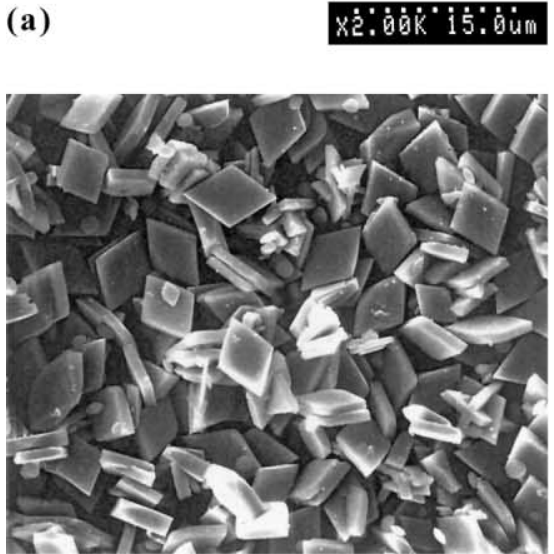

(b)
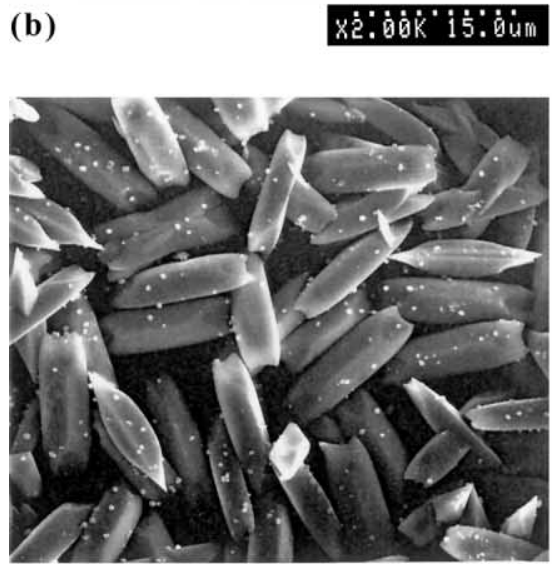

(c)

หล̉.

Fig. 7. Scanning electron micrographs of the $\mathrm{CeO}_{2}$ powders obtained by heating $\mathrm{CeOHCO}_{3}$ powders which are prepared using urea concentrations equal to (a) $0.05 \mathrm{M}$; (b) $0.1 \mathrm{M}$; (c) $1.0 \mathrm{M}$. 
prismatic form, and the size increases from 3-4 to $12-13 \mu \mathrm{m}$. The crystallinity of obtained $\mathrm{CeO}_{2}$ powders is regarded to be regulated by the particle size of $\mathrm{CeOHCO}_{3}$ powders. As expressed in Eq. (3), when $\mathrm{CeOHCO}_{3}$ transforms into $\mathrm{CeO}_{2}$, the $\mathrm{CO}_{2}$ gas is simultaneously generated. For large $\mathrm{CeOHCO}_{3}$ particles obtained at high concentration of urea, it is more difficult for the $\mathrm{CO}_{2}$ gas to dissipate from the interior region to the exterior. Therefore, the phase transformation from $\mathrm{CeOHCO}_{3}$ to $\mathrm{CeO}_{2}$ is not kinetically favored in the large $\mathrm{CeOHCO}_{3}$ particles, and the crystallinity of derived $\mathrm{CeO}_{2}$ powders is decreased. It is concluded that the particle size of $\mathrm{CeOHCO}_{3}$ plays an important role in modulating the crystallinity and the particle size of $\mathrm{CeOHCO}_{3}$ plays an important role in modulating the crystallinity and the particle size of $\mathrm{CeO}_{2}$.

\section{Conclusions}

Pure $\mathrm{CeOHCO}_{3}$ powders with high crystallinity are successfully fabricated via the newly developed hydrothermal process at $160^{\circ} \mathrm{C}$. Urea concentration shows significant effects on the morphology of $\mathrm{CeOHCO}_{3}$ powders. The hydrothermally obtained powders exhibit rhomboidal or prismatic shapes by modulating the concentration of urea solutions. Higher urea concentrations result in larger particle size as well as greater aspect ratio of $\mathrm{CeOHCO}_{3}$ powders. Cubic $\mathrm{CeO}_{2}$ is obtained via heating the hydrothermally derived $\mathrm{CeOHCO}_{3}$ powders at $500^{\circ} \mathrm{C}$. Fine $\mathrm{CeO}_{2}$ particles with high crystallinity are formed by heating fine $\mathrm{CeOHCO}_{3}$ powders.

\section{Acknowledgments}

The authors would like to thank Dr. R. Jagannathan for his valuable opinions and suggestions.

\section{References}

[1] E. Verdon, M. Devalette, G. Demazeau, Mater. Lett. 25 (1995) 127.

[2] P.L. Chen, I.W. Chen, J. Am. Ceram. Soc. 76 (1993) 1577.

[3] Y.C. Chou, R.J. Phillips, J.A. Switzer, J. Am. Ceram. Soc. 78 (1995) 981.

[4] X. Chu, W.I. Chung, L.D. Schmidt, J. Am. Ceram. Soc. 76 (1993) 2115.

[5] N.B. Kirk, J.V. Wood, J. Mater. Sci. 30 (1995) 2171.

[6] M.I. Diaz-guemes, T.G. Carreno, C.J. Serna, J.M. Palacios, J. Mater. Sci. Lett. 7 (1988) 671.

[7] T. Kanawaku, K. Amezawa, N. Yamamoto, Solid State Ionics 133 (2000) 51.

[8] M. Hirano, E. Kato, J. Am. Ceram. Soc. 82 (1999) 786.

[9] Y.C. Chou, M.N. Rahaman, J. Mater. Res. 8 (1993) 1680.

[10] C.-H. Lu, W.J. Huang, Mater. Lett. 27 (1996) 229.

[11] C.-H. Lu, S.Y. Lo, Mater. Res. Bull. 32 (1997) 371.

[12] C.-H. Lu, W.J. Huang, Mater. Lett. 27 (1996) 229.

[13] C.-H. Lu, W.C. Lee, S.J. Liou, G.T.K. Fey, J. Power Sources 81/82 (1999) 696. 
[14] E. Matijevic, W.P. Hsu, J. Colloid Interface Sci. 118 (1987) 506.

[15] W.P. Hsu, L. Ronnquist, E. Matijevic, Langmuir 4 (1988) 31.

[16] E. Matijevic, Chem. Mater. 5 (1993) 412.

[17] Powder Diffraction File, Card no. 41-13, Joint Committee on Powder Diffraction Standards, Swarthmore, PA, USA.

[18] E. Matijevic, Acc. Chem. Res. 14 (1981) 22.

[19] Powder Diffraction File, Card No. 34-394. Joint Committee on Powder Diffraction Standards, Swarthmore, PA, USA. 